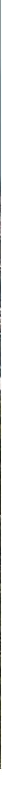

\title{
La Tierra Vacante en la ciudad de Xalapa. Una mirada desde la expansión urbana
}

\section{Pedro Martínez Olivarez - Arturo Velázquez Ruiz - Ma. Gpe. Noemi Uehara Guerrero ${ }^{1}$}

RESUMEN: El presente trabajo habla sobre el suelo vacante de la ciudad de Xalapa, sus orígenes, características e impactos de este, y una reflexión sobre su posible gestión para propiciar una ciudad compacta.

Palabras clave: Tierra Vacante, densidades, ciudad compacta, política de suelo.

\footnotetext{
${ }^{1}$ Dr. Arq. Pedro Martínez Olivarez, Universidad Veracruzana, Facultad de Arquitectura Xalapa, contacto: alarife76@gmail.com / pemartinez@uv.mx; Mtro. Arq. Arturo Velázquez Ruiz, Universidad Veracruzana, Facultad de Arquitectura Xalapa, contacto: arq.arturo.vr@gmail.com / arvelazquez@uv.mx; Dra. Arq. Ma. Gpe. Noemí Uehara Guerrero, Universidad Veracruzana, Facultad de Arquitectura Xalapa, contacto: guehara@uv.mx
} 
ABSTRACT: The present work deals with the vacant land of the city of Xalapa, its origins, characteristics and impacts, ending with a reflection on its possible management to achieve a compact city.

Keywords: Vacant Lots, Densities, Compact Cities, Land Policy

\author{
Introducción
}

L

a ciudad de Xalapa localizada en el estado de Veracruz, México, es una urbe que considera en su área conurbada a los municipios de Banderilla, Emiliano Zapata, Tlalnelhuayocan y Xalapa. Es una de las 59 zonas metropolitanas del país definidas a partir de una conurbación intermunicipal o interestatal (INEGI, CONAPO y SEDESOL, 2012), sin embargo, a esa delimitación, habrá que agregar los municipios de Coatepec, Jilotepec y Rafael Lucio, no considerados en la conurbación, pero sí en la región urbana metropolitana. Habiendo mencionado estos criterios de delimitación como marco de referencia espacial, diremos que, para efectos de este estudio, sólo se ha considerado la mancha urbana correspondiente al municipio de Xalapa, en términos prácticos y con excepción de Banderilla, Emiliano Zapata y Tlalnelhuayocan, el resto de las localidades todavía no se encuentran fusionadas mediante lo que podríamos definir como suelo continuo de destino urbano.

Otros hechos para atender sólo al municipio de Xalapa tienen que ver con el sentido de prevalencia de una ciudad central respecto al sistema de localidades, así como la implementación de políticas urbanas, donde si bien existen programas urbanos estatales que atienden los mantos territoriales de la conurbación, en la práctica los municipios siguen sus propias políticas de gestión del suelo, por lo que atenderemos sólo los estudios relacionados con Xalapa.

A partir de esta serie de consideraciones, podemos empezar a trabajar el tema de la Tierra Vacante (TV) en el área urbana municipal de Xalapa, con 457,928 habitantes y, que según estudios como el Informe Final Municipal 2016 (BID, BANOBRAS y AYUNTAMIENTO, 2014), en los últimos 35 años presenta un ritmo de crecimiento de la mancha urbana mayor al de la población, lo cual está generando una disminución en su densidad urbana, pasando de 9,293 hab/km² en 1980 a 7,041 hab/km² en 2015, un hecho todavía positivo si tomamos en cuenta que el promedio de densidad urbana en el país ronda los 5,000 hab $/ \mathrm{km}^{2}$, lo cual habla de un 
Artículo - Observatorio Urbano Universitario

cierto sentido de resistencia a la ciudad dispersa, por lo que cabe preguntarse ¿A qué se debe entonces ese crecimiento urbano actual extremadamente expansivo?

La Tierra Vacante desde la expansión xalapeña.

Hablar de la TV en la ciudad de Xalapa implica hacer un recorrido sobre las formas de producción del espacio y los patrones de crecimiento, principalmente a partir de la segunda mitad del siglo XX, el Plan de acción Xalapa Sostenible hace un muestreo donde se identifica un patrón de crecimiento urbano definiendo una lógica de comercio de la tierra como la evolución del uso y destino del suelo (BID, BANOBRAS y AYUNTAMIENTO, 2014), sin embargo, esta evolución no podría entenderse sin un hecho particular: la prevalencia de un direccionamiento del crecimiento urbano fincado en el eje de camino y las rutas de salida del centro a la periferia, particularmente sobre el eje carretero México-Veracruz (hoy Av. Lázaro Cárdenas) y que en gran parte explica el crecimiento urbano de la ciudad con respecto a su demarcación municipal y poblados conurbados (Figura 1).

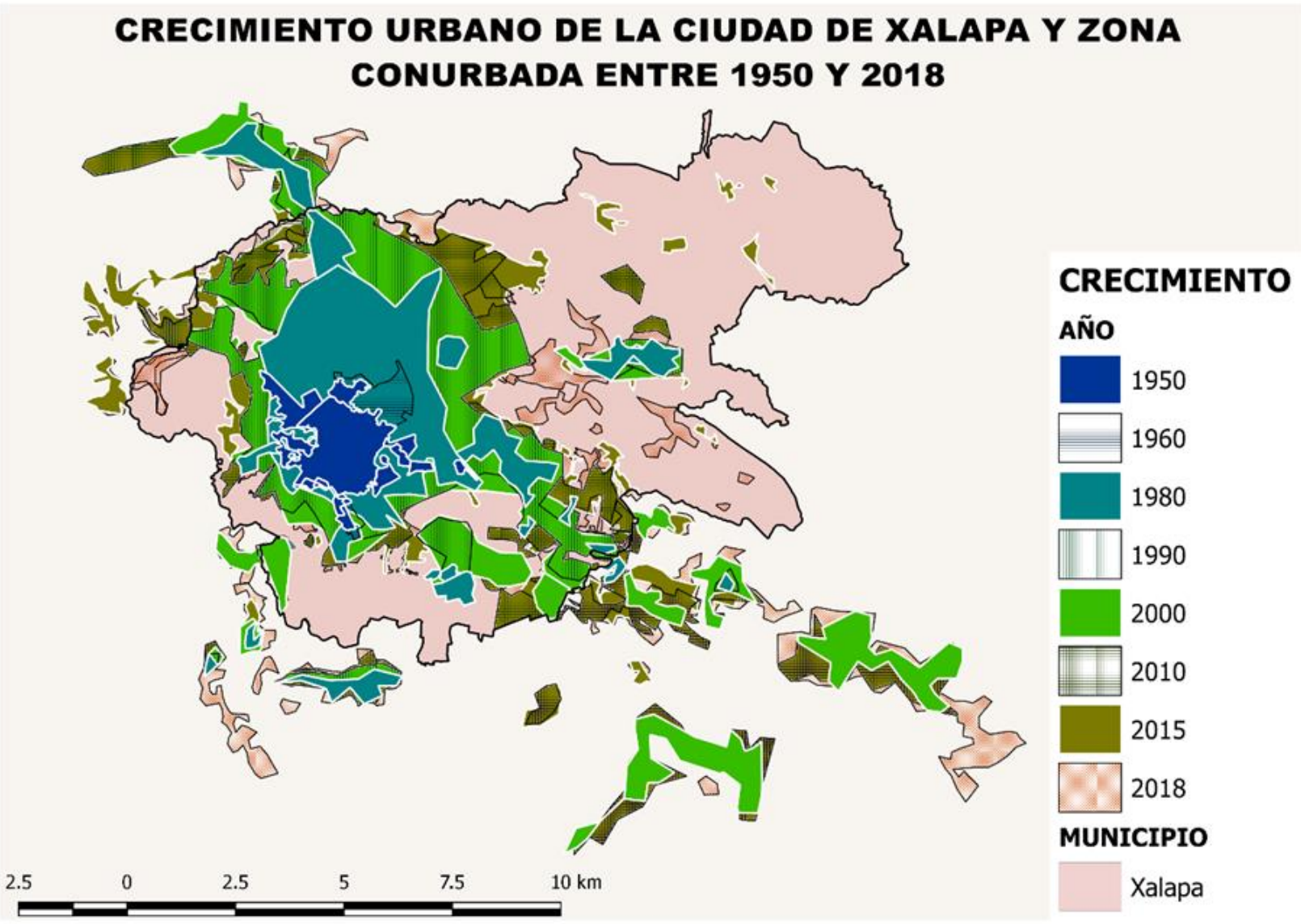




\section{Artículo - Observatorio Urbano Universitario}

Figura 1. Crecimiento urbano de la ciudad de Xalapa y zona conurbada entre 1950 y 2018. Fuente: Elaboración propia. Autor: Pedro Martínez Olivarez. Dibujo: Víctor Zarcillo.

La ciudad de Xalapa se caracteriza por su generosidad, en cuanto al espacio público y áreas verdes respecto a otras ciudades de México. En el primer aspecto existen 42 hectáreas por cada 100,000 habitantes y en el segundo 130 hectáreas por cada 100,000 habitantes (BID, BANOBRAS y AYUNTAMIENTO, 2014), todo esto da una idea de la calidad de la vida urbana de sus habitantes debido a la existencia de zonas de integración social, refleja también la paradoja entre la expansión y la densificación, ya que el hecho de contar con estas extensiones de espacio público tanto recreativo como ecológico, implica el ensanchamiento urbano y en cierta medida, un perfil predominantemente horizontal de escasa verticalidad que en cierto sentido, es contradictorio a la ciudad compacta.

La ciudad de Xalapa, se caracteriza por su generosidad, en cuanto al espacio público y áreas verdes respecto a otras ciudades de México. En el primer aspecto existen 42 hectáreas por cada 100,000 habitantes y en el segundo 130 hectáreas por cada 100,000 habitantes (BID, BANOBRAS y AYUNTAMIENTO, 2014), todo esto da una idea de la calidad de la vida urbana de sus habitantes debido a la existencia de zonas de integración social, refleja también la paradoja entre la expansión y la densificación, ya que el hecho de contar con estas extensiones de espacio público tanto recreativo como ecológico, implica el ensanchamiento urbano y en cierta medida, un perfil predominantemente horizontal de escasa verticalidad que en cierto sentido, es contradictorio a la ciudad compacta.

Esta es la densificación relacionada con la producción del espacio urbano surgida a partir de 1980, cuando se presentaban escasos vacíos inmobiliarios en el centro; ${ }^{2}$ hacia un perfil urbano horizontal que se arraiga en la idea sobre la propiedad privada; ${ }^{3}$ y la consecuente expansión fincada en el uso y destino del suelo, es decir, no siempre concretada en lo físico sino en la relación del ejercicio de sujeción especulativa de la propiedad. Además de las

\footnotetext{
${ }^{2}$ Cabe aclarar que es en 1980 cuando se emite el primer Plan de Desarrollo Urbano para Xalapa, que, sustentando gráficamente en una sectorización, empieza a hacer mella en las formas de producción espacial concéntricas, es decir, se pasa de direccionamientos expansivos relacionados con el viejo centro, a nuevos direccionamientos relacionados con el uso y destino siguiendo ejes de camino, y que por lo tanto, podemos afirmar, modificarían en parte la relación extensión-población traducida como densificación.

${ }^{3} \mathrm{Al}$ respecto, en el Foro sobre Ciudad Compacta acontecido en el curso en línea Tierra Vacante del Instituto Lincoln en el año 2017, se mencionó que, en cierta medida, hay un probable momento de aceptación social de la ciudad dispersa y horizontal a partir del sentido de propiedad privada sobre una fracción de suelo, el cual, tiene relación con una ocupación a manera de inversión patrimonial: ¿Compro aire o compro tierra? ¿Verticalidad u horizontalidad? ¿Edificio o casa? En cierta medida, es esa idea privativa que prima sobre la propiedad, la que causa el problema expansivo de la ciudad.
}

UVserva No. 8 Octubre 2019 - marzo 2020, ISSN: 2448-7430.

Recepción: 16/enero/2019

Aceptación: 22/septiembre/2019

DOI: 
Artículo - Observatorio Urbano Universitario

abundantes áreas verdes y espacio público en los que por su cercanía se difuminan muchos límites entre la propiedad pública y privada no utilizada, también la desactualización y hermetismos del registro catastral, lo que en conjunto complica la identificación de la TV en la ciudad de Xalapa. Sin embargo, podemos afirmar que esa TV está predominantemente presente a manera de intervalos e intersticiales como consecuencia del crecimiento expansivo al sureste de la ciudad.

Haciendo eco a las definiciones morfológicas de TV de Fausto Brito (s/a) en el primer caso esos intervalos o interrupción en la continuidad del tejido urbano, son terrenos urbanos de configuración abierta separados por trozos de urbanización y que están en franca valorización por la infraestructura vial existente. En el segundo, los intersticiales son terrenos en zonas boscosas o rústicos agrícolas producto del desprendimiento de la propiedad ejidal en la zona de contención urbana, rodeados por fragmentos urbanos a manera de nuevas colonias periféricas y pequeños fraccionamientos que se van agregando a la ciudad que están siendo comercializados conforme se va dando su inclusión legal a las normas urbanas. En ambos casos están para el caso de Xalapa, tocando los límites del equilibrio ecológico considerando que el principal movimiento inmobiliario actualmente se desarrolla en el sureste de la ciudad.

Así, el hecho de ocupar la TV, en el caso de Xalapa, si bien resultaría benéfico para contrarrestar un modelo de ciudad dispersa todavía no patente del todo, implicaría en el caso de los intersticiales, la ocupación de zonas boscosas inmersas en el área de contención. Es en esta zona donde hay una constante presión inmobiliaria sobre un bosque mesófilo que de 2008 a 2012 ha perdido 97.6 hectáreas al año (BID, BANOBRAS y AYUNTAMIENTO, 2014). En el caso de los intervalos su ocupación parece más factible, ya que es tierra localizada sobre infraestructura vial consolidada. Sin embargo, un caso como el del eje Rébsamen-Arco Sur, son ejemplo del debate entre la pertinencia social de la conservación ecológica o la necesidad social de suelo urbanizable. En este lugar mucha de la propiedad privada oscila entre los límites de una zona actualmente declarada Reserva Ecológica, el Parque Natura y la Reserva Tejar - Garnica y que representa, el mayor pulmón dentro de la mancha urbana, a la espera del mejor momento de comercialización, pervirtiendo aún más el proceso de ocupación del suelo urbano.

\section{Análisis de los instrumentos existentes.}


La expansión urbana de Xalapa debe seguir un rumbo más allá del tendencial (BID, BANOBRAS y AYUNTAMIENTO, 2014). En este sentido, la TV debe actuar bajo una vocación inmobiliaria de intervención del Estado en cuestiones rentísticas y tributarias y como un elemento que se engarce con los distintos instrumentos jurídicos en el aspecto medioambiental. Desde esta perspectiva, vemos una normativa hoy día centrada en un planteamiento ecológico que resulta pertinente para la contención urbana en aras de un crecimiento sostenible (Tabla 1).

No podemos dejar de mencionar las distintas escalas de poder que conviven en el espacio urbano y además que la TV no sólo es presa de especulación inmobiliaria, se convierte también en objeto de competencia política donde priman los intereses de quien detenta su usufructo, por ejemplo, están las reservas territoriales bajo el poder estatal dentro de los territorios municipales.

Tabla 1.

Instrumentos existentes y su incidencia en la sostenibilidad y la Tierra Vacante

\begin{tabular}{|c|c|l|l|}
\hline INSTRUMENTO & $\begin{array}{c}\text { ÁMBITOY } \\
\text { VIGENCIA }\end{array}$ & \multicolumn{1}{|c|}{ INCIDENCIA } \\
\hline \multirow{2}{*}{$\begin{array}{c}\text { Plan Veracruzano de } \\
\text { Desarrollo Región } \\
\text { Xalapa }\end{array}$} & Estatal & $\begin{array}{l}\text { En el apartado "Redimensionar la expresión } \\
\text { territorial del progreso" se habla de la necesidad de } \\
\text { "diseñar e implementar políticas públicas que } \\
\text { regulen el crecimiento y la reestructuración de los } \\
\text { centros de población urbanos y rurales, la calidad } \\
\text { de los servicios públicos con criterios de } \\
\text { sustentabilidad y prevención de riesgos, para } \\
\text { mejorar la calidad de vida de la población" } \\
\text { (Gobierno del Estado, 2016: 38). }\end{array}$ \\
\hline Plan Municipal de & Municipal \\
Desarrollo & 2014-2017 & $\begin{array}{l}\text { En las líneas estratégicas de acción se trata el } \\
\text { aspecto sustentable donde se dice que "Aunque en } \\
\text { Xalapa aún existe un importante conjunto de áreas } \\
\text { verdes, el desarrollo urbano está desplazando } \\
\text { rápidamente varios de los predios que se } \\
\text { constituían como pulmones de la ciudad. (...) La } \\
\text { ausencia de un programa de ordenamiento } \\
\text { ecológico del territorio, su implementación y }\end{array}$ \\
\hline
\end{tabular}

UVserva No. 8 Octubre 2019 - marzo 2020, ISSN: 2448-7430. 


\begin{tabular}{|c|c|c|}
\hline & & $\begin{array}{l}\text { cumplimiento ha provocado un crecimiento urbano } \\
\text { desordenado, que genera un impacto negativo al } \\
\text { medio ambiente. (H. Ayuntamiento, 2014: 81). }\end{array}$ \\
\hline INSTRUMENTO & $\begin{array}{l}\text { ÁMBITO Y } \\
\text { VIGENCIA }\end{array}$ & INCIDENCIA \\
\hline $\begin{array}{l}\text { Actualización del } \\
\text { Programa de } \\
\text { Ordenamiento } \\
\text { Urbano de la Zona } \\
\text { Conurbada Xalapa- } \\
\text { Banderilla-Coatepec- } \\
\text { Emiliano Zapata- } \\
\text { Tlalnelhuayocan. }\end{array}$ & $\begin{array}{l}\text { Conurbación } \\
\text { Desde } 2003\end{array}$ & $\begin{array}{l}\text { En el apartado del Nivel Estratégico de } \\
\text { implementación, se menciona cómo "la ciudad de } \\
\text { Xalapa crece en su continuo hacia el sudeste, pero } \\
\text { ahora siguiendo un patrón donde se alternan de } \\
\text { forma equilibrada las zonas de poblamiento con la } \\
\text { presencia constante y significativa de áreas } \\
\text { naturales protegidas y restauradas". Se habla } \\
\text { también de la posición en esta zona de las bolsas de } \\
\text { suelo aportadas por el municipio de Emiliano } \\
\text { Zapata al de Xalapa, al ser el primero el de mayor } \\
\text { aptitud para proveer de suelo a la expansión } \\
\text { urbana, constituyéndose así el direccionamiento de } \\
\text { la ciudad central sobre este municipio conurbado } \\
\text { (Gobierno del Estado, 2003: s/p). }\end{array}$ \\
\hline $\begin{array}{l}\text { Ley de Desarrollo } \\
\text { Urbano, } \\
\text { Ordenamiento } \\
\text { Territorial y } \\
\text { Vivienda para el } \\
\text { Estado de Veracruz } \\
\text { de Ignacio de la } \\
\text { Llave. }\end{array}$ & $\begin{array}{c}\text { Estatal } \\
\text { Desde } 2016\end{array}$ & $\begin{array}{l}\text { En el artículo } 31 \text { referente a la prioridad de } \\
\text { constituir reservas territoriales, se dice en el } \\
\text { apartado III que se deberá "Asegurar la oferta de } \\
\text { suelo necesaria para el establecimiento de los } \\
\text { programas de vivienda, de infraestructura y de } \\
\text { equipamiento requeridos, para evitar la } \\
\text { especulación inmobiliaria" (Gobierno del Estado, } \\
\text { 2016: 38). }\end{array}$ \\
\hline
\end{tabular}

Fuente: Elaboración propia consultando los instrumentos referidos.

El caso gráfico de las formas jurídicas es abordado únicamente por el Programa de Ordenamiento Urbano de la Zona Conurbada, cuyo plano, muestra lo relatado hasta este momento: la intensificación expansiva al sur de la ciudad en el eje de salida al Puerto de Veracruz, y la presión urbana que se cierne sobre la Reserva Ecológica y el bosque mesófilo de contención urbana. Lo relevante en este plano son los patrones de densidad señalados para el uso de suelo habitacional, en las densidades A y MA principalmente, que 


\section{Artículo - Observatorio Urbano Universitario}

identificadas como altas y medias altas, son esfuerzos tendientes a una verticalidad habitacional que podemos decir hasta hoy, que no se ha concretado.

Si bien en términos normativos, la TV está contemplada desde su pertinente ocupación como elemento incentivo de compactación urbana, por el contrario, desde la política del uso y destino del suelo, actúa coercitivamente al convertirse en un espacio fluctuante entre la concreción física de infraestructura y la expectativa expansiva, lo cual, podemos decir alienta las intenciones especulativas de los tenedores de la tierra en razón del privilegio de la inversión pública, misma que no alienta el desprendimiento de la tierra y deja su desarrollo para el mejor momento de venta (Figuras 2 y 3).
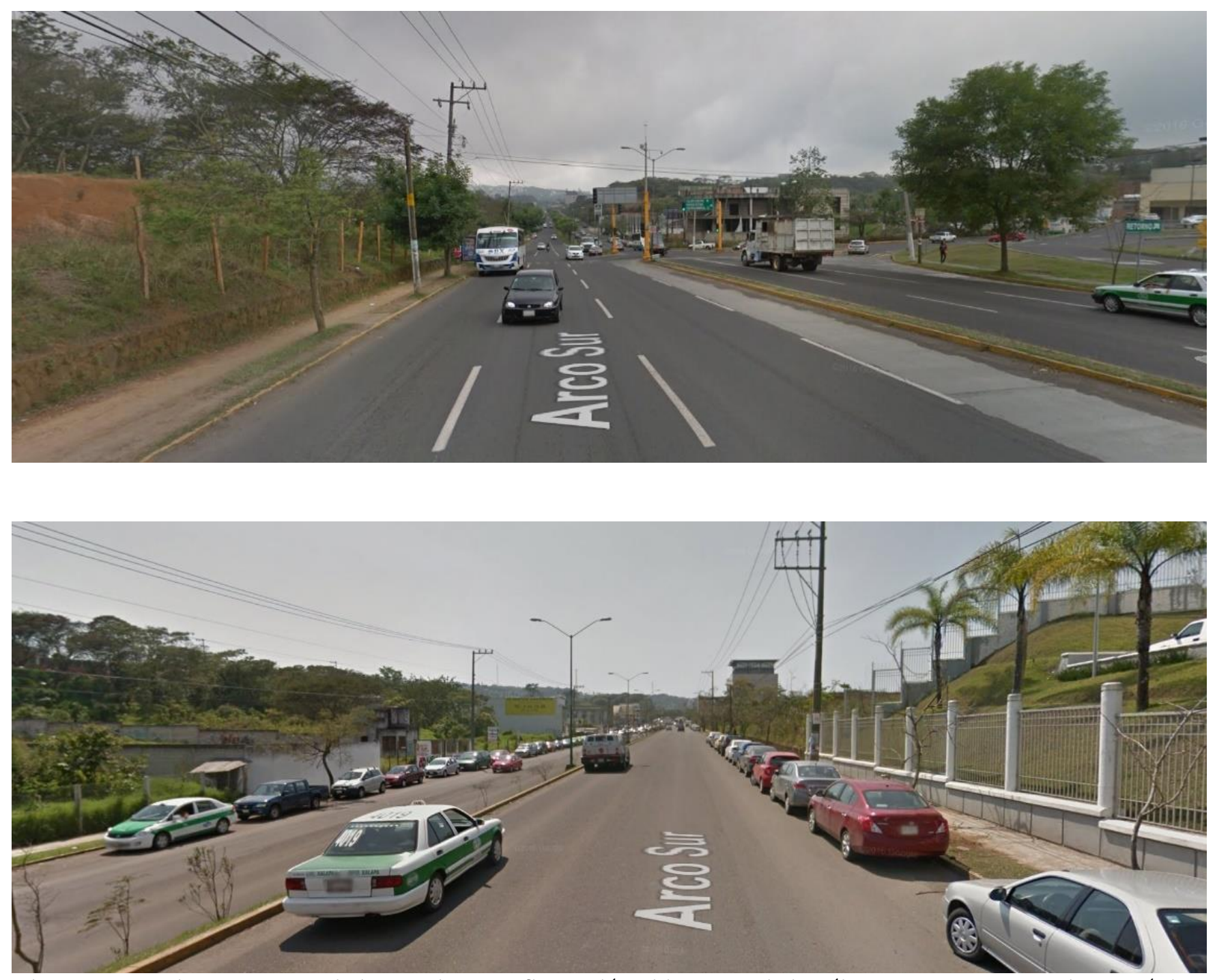

Figuras 2 y 3. Tierra Vacante de intervalo y configuración abierta en el eje Rébsamen-Arco Sur. Xalapa, México. Fuente: Google Maps (2018)

UVserva No. 8 Octubre 2019 - marzo 2020, ISSN: 2448-7430.

Recepción: 16/enero/2019

Aceptación: 22/septiembre/2019

DOI: 


\title{
Artículo - Observatorio Urbano Universitario
}

Al realizar el análisis de los instrumentos referidos en la Tabla 1, podemos observar que hay un reconocimiento normativo de la TV como un elemento utilitario espacial. Es principalmente el Programa de Ordenamiento Urbano de la Zona Conurbada el que hace mención de la TV. En este instrumento, se reconoce la conveniencia de utilización de la TV manteniendo además una constante de caracterización urbana en Xalapa: respecto a la preocupación por la saturación del espacio urbano, dice el programa:

\begin{abstract}
Deberá iniciarse la liberación de asentamientos humanos y recuperación de espacios para áreas verdes (...) además de establecer el control para evitar su ocupación con fines habitacionales en las zonas vacantes. / Debido a la existencia aún de algunas superficies de terreno vacante con posibilidades de ocupación con fines urbanos se proponen acciones de redensificación que aprovechen las redes de infraestructura y vialidad (...) / También se detecta la existencia de amplias zonas con terrenos vacantes que manifiestan posibilidades para admitir la incorporación de nuevos desarrollos habitacionales. / Zona con gran potencial para ser sometida a política de redensificación y saturación de predios actualmente vacantes, particularmente hacia el Sur de la Av. Rébsamen (...) / Dada su proximidad con el Centro Urbano y la disponibilidad de suelo vacante es factible de incorporar (...) espacio para la constitución de destinos alternos (...) a equipamiento urbano (Gobierno del Estado, 2004: s/p)
\end{abstract}

Es el mismo instrumento normativo el que aborda el papel de la TV en la construcción de densidades. En este sentido podemos relatar que a pesar de que se han establecido los mecanismos de ocupación tendientes a la redensificación, como el uso y destino, es poco el impacto de las edificaciones verticales en términos habitacionales; de no ser por las escasas unidades habitacionales, el predominio de la vivienda unifamiliar se haría más patente. Así, el programa dicta lo siguiente:

En este apartado (se refiere al de Densidades) se retoman los datos (...) que permitieron el reconocimiento (...) del comportamiento poblacional traducido al espacio urbano, identificando los diferentes grados de saturación presentes (...) y la correlación de estos parámetros de distribución con respecto a las posibilidades de asimilación de población nueva, atendiendo en primera instancia a la disponibilidad de suelo vacante. / Densidad muy alta. (...) el criterio seguido para la aplicación de este rango se basa en una política de impulso y fomento de los espacios vacantes con posibilidades de redensificación (...) se permitirá la posibilidad de incrementar la relación de viviendas por unidad de superficie en los predios vacantes (...) (Ídem.)

\section{Cuantificación y propuestas sobre la tierra vacante.}




\section{Artículo - Observatorio Urbano Universitario}

Los datos duros oficiales no indican cuál es la superficie de TV en Xalapa. Existe un indicador del estudio hecho por el Banco Interamericano de Desarrollo donde se da una superficie de 716 hectáreas aptas de urbanización (BID, BANOBRAS y AYUNTAMIENTO, 2014), pero esa área, responde por un lado a la tendencia expansiva, y por el otro, a una delimitación urbana engranada con el agrupamiento municipal, que como hemos señalado al inicio, difiere dependiendo de la escala de observación, es decir, si de lo que se habla es de una conurbación o de una metropolización.

Sin embargo, no podemos dejar de señalar la importancia de ese estudio en cuanto a sostenibilidad para Xalapa a partir del señalamiento de la necesidad de un uso de suelo de escenario inteligente, donde se integra la ciudad al territorio estableciendo limitantes para las diferentes áreas de desarrollo, ello, con la consideración de perspectivas ambientales integradoras de corredores viales y espacios verdes que además posibiliten la diversificación de actividades (BID, BANOBRAS y AYUNTAMIENTO, 2014).

Esa necesidad de corregir la tendencia expansiva, puede dar pauta a las propuestas sobre la TV para el caso de Xalapa, antes, identificaremos y cuantificaremos lo que creemos es el suelo urbano sin utilizar en la ciudad de Xalapa. Hemos hablado hasta este momento de la TV de intervalo y configuración abierta (intersticios), sin embargo, en la ciudad, existen innumerables huecos y perforaciones, es decir, construcciones vacantes desocupadas y lotes baldíos dispersos a manera de suelo atomizado (Fausto Brito, s/a) que en conjunto con os intervalos representan un aproximado de 134 hectáreas de reserva de suelo exponencialmente utilizable (Figura 4).

Esta cuantificación fue realizada identificando mediante el plano de usos y destinos del suelo, los lotes señalados en el mismo como baldíos y los que de acuerdo al mismo registro, se encuentran en desuso. Es evidente que habrá que hacer un análisis más detallado al respecto, sin embargo, haciendo un ejercicio de relación, esas 134 hectáreas que arroja nuestro estudio representan el $20.42 \%$ respecto a las 656 hectáreas de mancha urbana actual de Xalapa, es decir, una quinta parte del continuo urbano está en desuso. La misma superficie de TV es un $18.71 \%$ respecto a las 716 hectáreas aptas de urbanización que menciona el Banco Interamericano de Desarrollo, es decir que, con su ocupación, podría ahorrase casi una quinta parte del suelo de expansión contribuyendo a la ciudad compacta.

Mucho se habrá que decir sobre las causas de este vacío urbano. Una de ellas podría ser las condiciones topográficas, que además de dificultar y encarecer la construcción, interviene en la identificación y censo de la TV. Al respecto, la ciudad de Xalapa tiene una variación de 
altitudes considerable por lo que el perfil horizontal en la vivienda no resulta monótono como en otras ciudades llanas, y es gracias a las pendientes que caracterizan a la ciudad, que se alienta la preservación de las áreas verdes

\section{TIERRA VACANTE EN LA CIUDAD DE XALAPA}

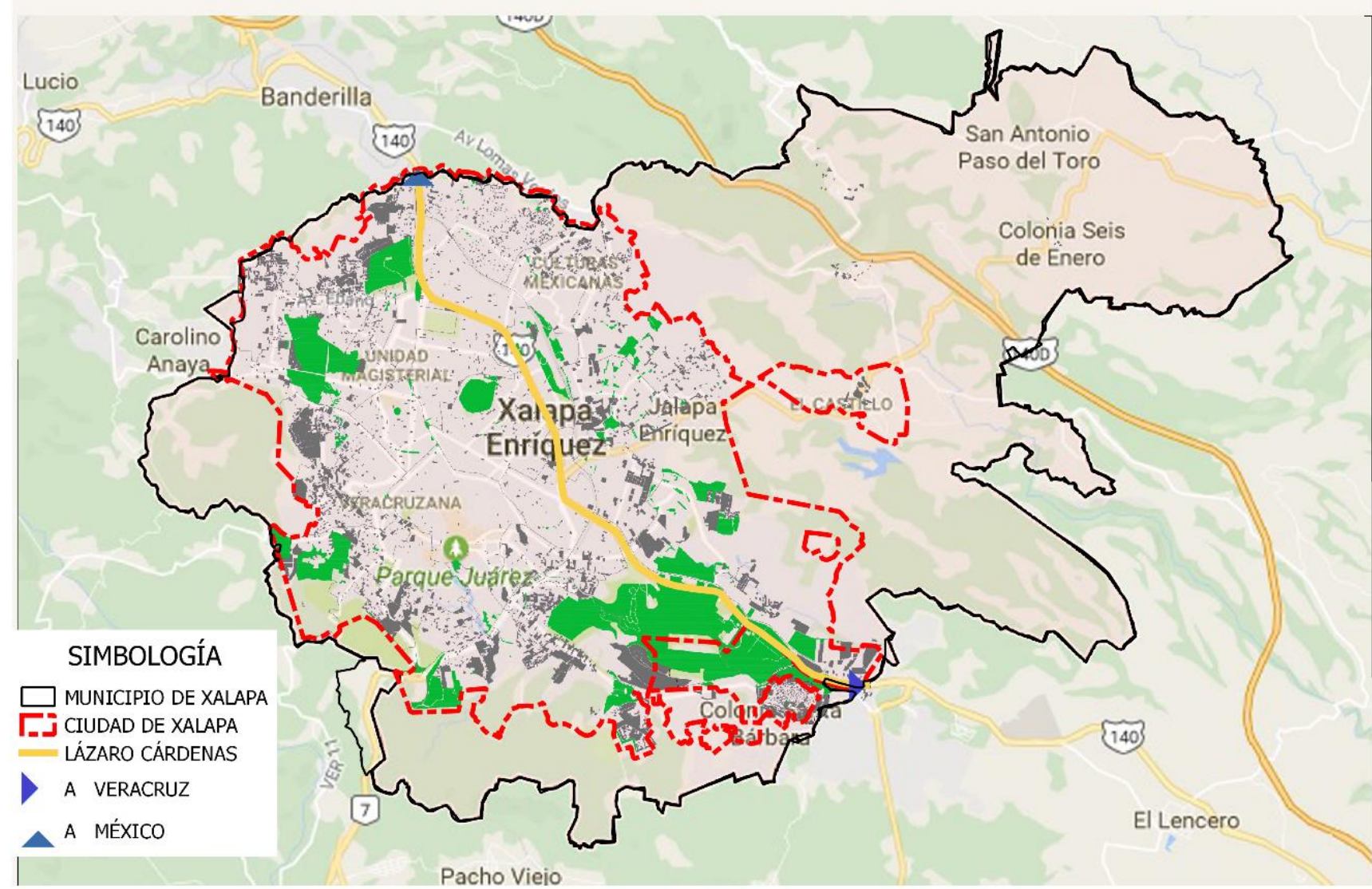

Figura 4. Plano de Tierra Vacante y áreas verdes en el municipio de Xalapa. Fuente: Elaboración propia. Autor: Pedro Martínez Olivarez. Dibujo: Víctor Zarcillo.

Desde estas perspectivas, creemos que las mejores propuestas en torno a la utilización de la TV, deben estar relacionadas con la caracterización de la misma. Primero proponemos que, para el caso de los huecos, identificados principalmente en el centro de la ciudad, deben considerarse proyectos de revitalización que mitiguen el deterioro arquitectónico, el despoblamiento del área central, y el desplome económico de la zona, en gran parte el Programa de Ordenamiento y Revitalización del Centro Histórico de Xalapa ya está atendiendo esta problemática (Gobierno del Estado, 2005). Para el caso de las perforaciones, es decir, los lotes baldíos dispersos, creemos que la mejor forma de alentar su 
desprendimiento y aletargamiento funcional sería mediante un aumento del impuesto predial progresivo, que desincentive la tenencia especulativa sobre las fracciones individuales de suelo urbano, lo mismo para el caso de los intervalos, hoy día principales captadores de la plusvalía por nuevas infraestructuras y en los que debe alentarse la ocupación vertical en vivienda de tipo mixto. Así, la mejor propuesta para colocar en el mercado la TV sería la actualización inmediata de toda la normativa inmersa en el uso, destino, registro y tenencia de la tierra, para de esa manera, concretar la compactación y sostenibilidad urbana.

\section{Conclusiones}

A lo largo del texto se han señalado las características del crecimiento urbano de la ciudad de Xalapa en relación con el perfil urbano y un modo de producción del espacio en el que se alienta la provisión de espacios recreativos para la construcción del tejido social y la preservación del medioambiente, esto es paradójico considerando la actual ocupación inmobiliaria sobre suelo de conservación, pero descifrable desde la necesidad social de la vivienda que mantiene una constante lucha espacial entre lo rural y lo urbano.

Esta tendencia ecológica impresa tanto en las normas como en la conciencia social, da a Xalapa un carácter de sostenibilidad en el que el factor medioambiental al parecer se encuentra desarrollado, sin embargo, la situación de la TV en el que se observa la gran pulverización del suelo en torno a la tenencia por la individualización de la propiedad contrastando con las superficies extensas disponibles en los ejes expansivos, dan cuenta de un desacople con el factor urbano que retarda la pretendida sostenibilidad.

Así, la ciudad de Xalapa, a partir de la expansión urbana acontecida, no es producto de una predominancia horizontal por concreción física, sino que hay una expansión fincada sobre la ocupación del territorio mediante los usos y destinos del suelo que involucran atomización de la tierra y existencia de TV no dinamizada. Por lo que la TV es en Xalapa, más que un problema, una oportunidad para una ciudad que hoy todavía es manejable en cuanto a escala en el campo de la planificación, todo, en aras de dar respuesta a los tres aspectos que la consoliden como ciudad compacta: ambiental, urbano y económico. 
Artículo - Observatorio Urbano Universitario

\section{Referencias}

BID, BANOBRAS y AYUNTAMIENTO (2014) Plan de acción Xalapa sostenible: visión para un futuro con servicios eficientes, un territorio resiliente y cuentas transparentes. Banco Interamericano de Desarrollo-Banco Nacional de Obras y Servicios Públicos-H. Ayuntamiento de Xalapa. Xalapa, Ver.

Fausto Brito, A. (s/a) Conceptos relacionados con los espacios vacantes en la ciudad. Centro de Estudios Metropolitanos. CUAAD. UdeG.

Gobierno del Estado. (19 de marzo de 2004) Actualización del Programa de Ordenamiento Urbano de la Zona Conurbada Xalapa-Banderilla-Coatepec-Emiliano ZapataTlalnelhuayocan, Ver. Gaceta Oficial del Gobierno del Estado de Veracruz de Ignacio de la Llave.

Gobierno del Estado. (2005) Programa de Ordenamiento y Revitalización del Centro Histórico de Xalapa, Ver. Secretaría de Desarrollo Regional-Gobierno del Estado de Veracruz de Ignacio de la Llave.

Gobierno del Estado. (Enero de 2016). Ley de Desarrollo Urbano, Ordenamiento Territorial y Vivienda para el Estado de Veracruz de Ignacio de la Llave. Secretaría de Gobierno.

Gobierno del Estado. (29 de noviembre de 2016). Plan Veracruzano de Desarrollo 2016-2018 Región Xalapa. Gaceta Oficial del Gobierno del Estado de Veracruz de Ignacio de la Llave.

H. Ayuntamiento. (2014) Plan Municipal de Desarrollo 2014-2017. El Colegio de Veracruz. Xalapa, Ver.

INEGI, CONAPO y SEDESOL (2012) Delimitación de las zonas metropolitanas de México 2010. Instituto Nacional de Estadística y Geografía-Consejo Nacional de PoblaciónSecretaría de Desarrollo Social, Aguascalientes, Ags. - México, D.F. 